Ovarian cancer is one of the most prevalent pathologies in gynaecology. This malignancy can be divided into 2 large groups: epithelial and non-epithelial. Because epithelial ovarian cancers (EOC) are the most commonly diagnosed, this paper focuses on the latest therapies associated with this disease. Due to the difficult diagnosis, EOC is frequently detected in the advanced stage. The treatment is usually complex and requires specialist knowledge. Advances and new ideas, such as identification of various genes and molecules that can serve as prognostic factors, might increase patients' chances of survival; they may contribute to optimization of patients' treatment, deciding whether to use aggressive treatment strategies, and predicting chemoresistance. Moreover, new strategies might also improve the quality of life of patients. The study aimed to analyse and discuss the latest reports on new methods of managing EOC.

Key words: ovarian cancer, immunotherapy, chemotherapy, epithelial cancer, treatment.

Contemp Oncol (Pozn) 2021; 25 (4): 225-231 DOl: https://doi.org/10.5114/wo.2021.112037

\section{What is new about ovarian malignancies?}

\author{
Kinga Grabska', Izabela Pilarska ${ }^{1}$, Marta Magdalena Fudalej², \\ Andrzej Deptała², Anna Badowska-Kozakiewicz ${ }^{2}$
}

'Students' Scientific Organization of Cancer Cell Biology, Department of Cancer Prevention, Medical University of Warsaw, Poland 2Department of Cancer Prevention, Medical University of Warsaw, Poland

\section{Introduction}

Ovarian cancer is one of the most prevalent pathologies in gynaecology. The current histopathological classification distinguishes over 70 types of tumours that may be detected in the ovary. Ovarian tumours can be divided into 2 large groups: epithelial and non-epithelial. Most ovarian cancers are sporadic; only $5-10 \%$ of them are presented with a family history [1].

Ovarian cancer incidence is relatively stable in Western countries. However, the percentage of ovarian cancer among gynaecological neoplasms is increasing, which is related to the decline in the incidence of cervical cancer in European countries due to cytological screening programs [2]. Trends in relative survival and population mortality show slight improvement. The changes in mortality can be explained partially by a decrease in the incidence of ovarian cancer (presumably due to the use of oral contraceptives and a reduction in the use of hormones after menopause) [3].

Survival in ovarian cancer is the worst among all gynaecological cancer locations [4]. The main reasons for the poor survival include the lack of early detection methods and the ovaries' unfavourable anatomical location. Treatment for ovarian cancer is complex and requires specialist knowledge and experience in oncology and surgery [2, 5-7]. Advances in ovarian cancer treatment can significantly improve patients' chances of survival.

The study aimed to analyse and discuss the latest reports on new methods of managing epithelial ovarian cancer (EOC). Articles published from 1976 to 2020 in the PubMed and Elsevier databases were analysed. The authors focused on analysing risk factors, prognostic and predictive factors, and possible cancer therapies.

\section{Epithelial ovarian cancer - characteristics}

The most common type of ovarian cancer is epithelial, occurring in 95\% of cases [8]. Every year 220,000 women in the world are diagnosed with this malignancy [9]. The high death rate results from non-specific early symptoms, late diagnosis, and high metastatic rate within the abdomen. Despite modern management and complete response to the treatment, many patients diagnosed with advanced disease develop a recurrence within 2.5 years [10].

Four primary histological types of EOC can be distinguished: serous (high-grade serous carcinomas [HGSC] and low-grade serous carcinomas), endometrioid, mucinous, and clear cell. Epithelial ovarian malignancies are divided into 2 categories - type I and type II tumours. The first type comes originally from the ovary and is caused by continual ovulation cycles, endometriosis, and inflammation. These tumours are more often diagnosed as a disease of a low stage. Therefore, they are less lethal and usually present a better outcome than the second type, which comes usually from a fallopian tube and is related to genetic mutations of the BRCA gene, p53, or other tumour-suppressing genes [8]. 
Among EOC symptoms, the following might be distinguished: abdominal pain and distention, early satiety, constipation, bloating, nausea, signs from the urinary tract, later fatigue, and loss of weight [11]. The measurement of CA-125 concentration, pelvic ultrasound followed by pelvic computed tomography or magnetic resonance imaging, and diagnostic surgery with pathological examination are the initial diagnostic investigations. The first-line treatment of EOC consists of the primary debulking surgery and platinum-based chemotherapy with or without an anti-endothelial growth factor (VEGF) agent [12].

\section{What is new about epithelial ovarian cancer?}

\section{Risk factors}

Having a family history of breast and ovarian cancer, mutations of breast cancer type 1 susceptibility protein (BRCA 1), breast cancer type 2 susceptibility protein (BRCA 2), and DNA mismatch repair genes, uninterrupted ovulation cycles (early onset of menses, childlessness, late menopause), endometriosis, ethnicity, smoking, hormonal replacement therapy, and diet are well-known risk factors of the occurrence of EOC [8]. Although these endangering agents are well-known and widely studied, many studies about them and newly discovered factors are reported each year.

The latest studies have revealed that the gender of the offspring might also present an impact on EOC risk. Bearing a male offspring was associated with an $8 \%$ lower risk of EOC. Compared to bearing all-female offspring, having all-male offspring was associated with a $14 \%$ decrease in EOC risk. The protective effect seems to be enhanced by the increasing number of male children. The biological explanations for this phenomenon might be associated with different maternal hormone concentrations [13]. Despite the fact that the study failed to reach statistical significance, previous studies - in eastern Pennsylvania [14] and Sweden [15] - reported similar findings. Slightly different results were reported in a recent pooled analysis among participants from 12 case-control studies, which included 6872 EOC patients. It was found that each additional offspring was associated with an $8 \%$ decrease regardless of whether the child was male or female. The sex of the offspring was not linked with EOC risk for serous, clear cell, and endometrioid histotypes. However, bearing male offspring was associated with lower protection against mucinous histotype, but the male sex appeared to have no significant relation to this kind of EOC [16].

Modugno et al. showed in their research that breastfeeding for at least 3 months provides significant protection from EOC. This protection is increased by an earlier age at first breastfeeding and a larger number and longer duration of breastfeeding episodes. Although this protection decreases over time, it can last for more than 30 years [17]. Another study dealt with the protective value of breastfeeding among patients with BRCA mutations. It showed that breastfeeding history was associated with a decrease of $23 \%$ in ovarian cancer risk among BRCA carriers. From 1 to 7 months of breastfeeding increased the protective effect, and after that period the association was relatively stable. The use of oral contraceptives was report- ed to be a significant independent protective factor, which enhanced the positive effect of breastfeeding [18].

Other research dealt with the link between benign ovarian tumours and the risk of EOC. The cohort group consisted of over 150,000 women with a primary or secondary diagnosis of benign ovarian tumours (e.g. serous cystadenoma, mucinous cystadenoma, clear cell adenofibroma, benign Brenner tumour, thecoma, and fibroma) between 1978 and 2016. The occurrence of benign ovarian tumours doubly increased the risk of mucinous ovarian cancer. The risk was independent of the age and was accelerated up to 20 years after diagnosing benign ovarian tumours. No link was found between other histological types of EOC and previous benign ovarian tumours [19].

In the Ovarian Cancer Cohort Consortium study, a connection between tumour dominance and different reproductive and hormonal risk factors of EOC was observed. Women with a dominant tumour mass were less likely to be parous (among those who have fewer children), have ever smoked, have ever taken oral contraception (OC), have had a hysterectomy, tubal ligation, or unilateral oophorectomy, in comparison to women with a non-dominant tumour mass. Additionally, patients with a right-dominant tumour mass were more likely to be parous or to have ever used OC than those with a left-dominant tumour mass. The body mass index is related to a meaningly increased risk of left-dominant ovarian cancer. The linkage between the histological type of a tumour and dominance was also observed. There were more clear cell subtype masses in left-dominant cases and more serous subtypes of tumour in the right-dominant ones [20] (Table 1).

There are also some recent studies claiming that viral infections with Papillomavirus (HPV), Cytomegalovirus (CMV), and Epstein-Barr virus (EBV) [21], as well as an exposition to different types of asbestos fibres in occupational and environmental settings, may contribute to the development of ovarian cancers [22, 23].

\section{Prognostic and predictive factors}

The Federation of Gynaecology and Obstetrics (French. Fédération internationale de gynécologie et d'obstétrique - FIGO) staging system for ovarian cancer is the primary tool that provides prognostic information and guidance of management for ovarian malignancy. The assessment is based on diagnostic surgery and imaging [24]. Besides the FIGO staging, classifying EOC as types I or II, which depends on the histological type and grading, also implicates a prognosis for the patients. Type I is associated with better outcomes and more prolonged survival [25]. However, there are cases in which the disease progression does not correlate with the initial assessment. What else has an impact on the prognosis? The main reason for treatment failure of ovarian malignancy is resistance to platinum-based chemotherapy [26]. After platinum-based chemotherapy, many patients experience a relapse and become drug-resistant within 2 years. As a result, the survival rate for them is approximately $40 \%$ [27]. This phenomenon might be correlated with intrinsic or acquired factors and various ovarian cancer mechanisms [28]. Xing 
Table 1. Definition of dominant/non-dominant tumour mass [20]

Dominant tumour mass
Limited to one ovary
Tumour mass was found in one ovary, with only tumour foci on the
other ovary
Tumour dimensions, area, or volume on one side was at least twice
that of the other side

et al. analysed 6 genes [Fanconi anaemia complementation group A (FANCA), Fanconi anaemia complementation group G (FANCG), DNA Polymerase Delta 1 (POLD1), lysine (K)-specific demethylase 1A (KDM1A), Bloom syndrome protein (BLM), and BRCA 1], potentially affecting the answer to platinum-based treatment. The 6 candidate genes were verified in various validation sets. The insightful examination of these genes proved that the mRNA and protein expression levels possessed a meaningful difference in the analysis of clinical and pathological factors. Moreover, notable relationships between these genes and both overall survival and disease free survival were detected. The real-time polymerase chain reaction assay confirmed that those candidate genes' expression levels in platinum sensitive ovarian cancer cell lines were higher than those in platinum resistant ones [29].

There are also some specific molecules, expression of which can affect disease progression. Therefore, they can serve as a new prognostic marker and a therapeutic target for the EOC. The study of Zou et al. analysed the expression patterns, prognostic value, genetic variation, and biological functions of 12 members of the ubiquitin-conjugating enzyme E2 (UBE2) gene family. They observed that the mRNA levels of UBE2C, UBE2N, UBE2S, and UBE2T were notably upregulated in ovarian malignancy compared to normal ovarian tissue. Patients with serous ovarian cancer and upregulation of UBE2A, UBE2B, UBE2C, UBE2G, UBE2R2, and UBE2T were characterized by poor overall survival. Moreover, upregulation of UBE2A, UBE2N, and UBE2R2, and downregulation of UBE2T and UBE2G were associated with poor progression-free survival. Being significantly upregulated in EOC compared with that in borderline tumours, benign tumours, and normal ovarian tissues, UBE2T is thought to have a high diagnostic accuracy [30]. The study by Li et al. reported that UBE2C levels are significantly higher in cisplatin-resistant cells than in sensitive ones. Silencing of UBE2C expression using lentiviral-mediated short hairpin RNA (shRNA) in model cells resulted in enlarged sensitivity to cisplatin and increased apoptosis [31]. Another study proved that UBE2C and histone-lysine N-methyltransferase (EZH2) genes are potential therapeutic capture points and should be investigated for their clinical use [32].

Another prognostic and therapeutic target of EOC may be lysine-rich coiled-coil 1 (KRCC1). It is a nuclear protein overly expressed by tumour cells in patients with highgrade serous ovarian cancer. Higher levels of KRCC1 expression correlate with chemoresistance and poor outcomes. Silencing experimentally, KRCC1 inhibits cellular plasticity and invasive properties and enhances apoptosis, leading to tumour growth reduction [33].

\section{Non-dominant tumour mass}

Tumour was classified as primary peritoneal cancer

Only tumour foci were found on both ovaries, no ovaries could be identified on either side of the peritoneal cavity

Tumour dimensions, area, or volume on one side was within two times that of the other side

Some other genes and molecules may influence the EOC prognosis and contribute to treatment failure. Recent studies have concentrated on the following molecules (examples only): Annexin A8 (ANXA8) [34], Ten-Eleven Translocation Protein 3 (TET3) [35], Retinoic Acid-Inducible Gene-I (RIG-I) [36], Desmoglein-2 (DSG2) [37], Maternal Embryonic Lucine Zipper Kinase (MELK) [38], Eukaryotic Translation Initiation Factor 2B Subunit Epsilon (EIF2B5), B-Arrestin 2 [39], and RING Finger Protein 126 [40]. The potential of incorporating new molecules into prognostic and predicting schemes for ovarian cancer patients seem to be high; however, further, more complex research is needed.

\section{Treatment}

The primary treatment for early EOC is surgery. It is used for both staging and debulking (cytoreduction). It can be an exclusive and curative treatment in a disease confined to the ovaries. For later stages of the disease (IC and II), postsurgical chemotherapy is recommended. It usually consists of platinum and paclitaxel [41]. Platinum-based chemotherapy has been the primary management of ovarian cancer since the 1980s [3]. Neoadjuvant chemotherapy is also used in some nonoperative cases. Apart from intravenous chemotherapy, intraperitoneal can be ordered after suboptimal cytoreductive surgery in the FIGO III stage. Bevacizumab - a humanized monoclonal antibody targeting vascular VEGF - can be added to the conventional chemotherapy for patients after suboptimal cytoreductive (FIGO III stage) as well as for patients with disseminated carcinoma [42, 43]. Additionally, Olaparib - an oral poly (ADP-ribose) polymerase (PARP) inhibitor - can be ordered for patients with serous ovarian cancer and the presence of BRCA $1 / 2$ mutations. Olaparib serves as a maintenance treatment for patients after relapse, who responded to platinum-based chemotherapy [44].

Epithelial ovarian cancer therapy has barely changed in the last 40 years, and it still seems to lack effective therapeutic targets. Although EOC was supposed to be scarcely immunogenic, immunotherapy appears to have more therapeutic potential than was previously thought. A lot of emphases is placed on improving the management of ovarian cancer and making it more personalized [43]. So, what is new about the treatment of ovarian cancer?

The primary goal of surgical treatment is to accomplish a complete resection with clear margins. Score RO or R1 in the residual tumour (R) classification implicates better survival rates [45]. Ceppi et al. proved that targeted molecule-based fluorescence imaging helps achieve complete tumour resection on the microscopic scale. They used a fluorescence imaging system with an orthotopic mouse model 
Table 2. Examples of clinical trials involving the combination of immunotherapy and anti-angiogenic treatment in ovarian cancer

\begin{tabular}{|c|c|c|c|}
\hline Identifier & Agent/Drug & Study title & Status \\
\hline NCT02659384 & $\begin{array}{l}\text { Bevacizumab } \\
\text { Atezolizumab } \\
\text { Acetylsalicylic Acid }\end{array}$ & $\begin{array}{c}\text { Anti-programmed cell death-1 ligand } 1 \text { (aPDL-1) antibody atezolizumab, } \\
\text { bevacizumab and acetylsalicylic acid } \\
\text { in recurrent platinum resistant ovarian cancer }\end{array}$ & $\begin{array}{l}\text { Active, } \\
\text { not recruiting }\end{array}$ \\
\hline NCT03353831 & $\begin{array}{l}\text { Atezolizumab } \\
\text { Bevacizumab } \\
\text { Chemotherapy }\end{array}$ & $\begin{array}{l}\text { Atezolizumab with bevacizumab and chemotherapy vs. bevacizumab and } \\
\text { chemotherapy in early relapse ovarian cancer }\end{array}$ & Recruiting \\
\hline NCT03363867 & $\begin{array}{l}\text { Atezolizumab } \\
\text { Bevacizumab } \\
\text { Cobimetinib }\end{array}$ & $\mathrm{BEACON}-\mathrm{ABC}$ in recurrent platinum resistant $\mathrm{HGSOC}$ & Recruiting \\
\hline NCT04361370 & $\begin{array}{l}\text { Olaparib } \\
\text { Pembrolizumab } \\
\text { Bevacizumab }\end{array}$ & $\begin{array}{l}\text { Olaparib maintenance with pembrolizumab \& bevacizumab in BRCA } \\
\text { non-mutated patients with platinum-sensitive recurrent ovarian cancer }\end{array}$ & Not yet recruiting \\
\hline NCT03596281 & $\begin{array}{l}\text { Pembrolizumab } \\
\text { Bevacizumab } \\
\text { Pegylated } \\
\text { Liposomal } \\
\text { Doxorubicin }\end{array}$ & $\begin{array}{l}\text { Pembrolizumab in combination with bevacizumab and pegylated } \\
\text { liposomal doxorubicin in patients with ovarian cancer }\end{array}$ & Not yet recruiting \\
\hline NCT02853318 & $\begin{array}{l}\text { Pembrolizumab } \\
\text { Bevacizumab } \\
\text { Cyclophosphamide }\end{array}$ & $\begin{array}{c}\text { Pembrolizumab, bevacizumab, and cyclophosphamide in treating } \\
\text { patients with recurrent ovarian, fallopian tube, or primary } \\
\text { peritoneal cancer }\end{array}$ & $\begin{array}{l}\text { Active, } \\
\text { not recruiting }\end{array}$ \\
\hline
\end{tabular}

Data from ClinicalTrials.gov.

to assess tumour detectability and to evaluate the effect of fluorescence imaging-guided surgery. The contrast agent used in this study was an intra-peritoneal nanomolecular probe, composed of single-walled carbon nanotubes, coupled to an M13 bacteriophage carrying a modified peptide binding to the secreted protein acidic and rich in cysteine overexpressed in studied mice. High microscopic tumour detection was observed with a pixel-limited resolution of $200 \mu \mathrm{m}$. Additionally, the researchers observed an elevated survival in animals treated with fluorescence image-guided surgical resection compared to the typical surgery [46].

The pleiotropy of transforming growth factor-beta (TGF- $\beta$ ) signalling in tumour tissues covers cancer initiation, development, metastasis, and reaction between stroma and cancer cells [47]. TGF- $\beta$ plays a crucial role in the metastasizing by providing epithelial-mesenchymal transition (EMT). TGF- $\beta$ signalling may be activated by overexpression of domain-containing ion transport regulator 5 (FXYD5) in cancer cells. The TGF- $\beta$ activates the SMAD3/ SMAD4 complex, which initiates the transcription of further effectors and promotes FXYD5 transcription. The FXYD5 creates a positive loop with TGF- $\beta$ to drive EMT and, therefore, metastasis. It makes TGF- $\beta$ a potential target in the treatment of ovarian cancer [48]. It is claimed that the debulking signature is centred around the hyperactivation of the TGF- $\beta$ pathway, which drives the overexpression of genes located in the tumour and its microenvironment. Using the TGF- $\beta$ inhibitors with chemotherapy, for patients after suboptimal debulking surgery or in the neoadjuvant treatment, may improve the interval debulking surgery. TGF- $\beta$ inhibitors decrease migration and invasion of the tumour cells and increase response to the treatment [49]. Zhang et al. reported that inhibiting the TGF- $\beta$ pathway with LY2157299 monohydrate (TGF-R1 inhibitor) in animal models reduced tumour cell proliferation, migration, and invasion. Additionally, it was found that LY2157299 is in- volved in slowing down cancer growth and ascites formation. These effects are correlated with reduced expression of vital stroma proteins COL11A1 and VCAN [50].

In 2020, a study assessing the impact of prolonged preoperative cycles on survival, accounting for surgical outcomes, was published. The study compared the results of treatment of 199 women with newly diagnosed ovarian cancer. Women who received 3 or 4 neoadjuvant cycles were compared with women who received 5 or more cycles. Apart from the number of cycles between the groups of women, there were no other differences in clinical factors. The rates of complete resection were similar, regardless of the number of chemotherapy cycles received. Unfortunately, more cycles of chemotherapy (5 or more) were associated with poorer progression-free survival. The analysis showed that patients taking 3-4 cycles of chemotherapy had a better prognosis than patients receiving 5 or more cycles [51].

Some recent studies reported spontaneous tumour regressions [52, 53], occasional persistent responses to immune checkpoints-inhibitors [54], and longer progression-free time and overall survival in patients with tumours with a high rate of T-cells [55]. These revelations suggest that EOC patients would potentially benefit from immunotherapy.

Zamarin et al. showed that the combination of nivolum$a b$ and ipilimumab in platinum-resistant EOC resulted in better response and longer progression-free survival. However, more studies should be conducted [56].

Combining anti-angiogenic agents and immunotherapy might occur beneficially. The single usage of anti-angiogenic agents resulting in a reduced number of blood vessels and increased tumour hypoxia seems inadequate. However, immunotherapy might be supported by additional effects of drugs targeting VEGF, angiopoietin 2, or hepatocyte growth factor pathways. A few clinical trials 
evaluating immune-checkpoint inhibitors in combination with anti-angiogenic agents in patients with EOC are currently in progress [57] (Table 2).

PARP inhibitors (PARPis) have been used in EOC treatment since 2014, when the Food and Drug Administration and the European Medicines Agency approved them. Olaparib, Niraparib, Rucaparib, Talazoparib, and Veliparib are the most relevant PARPis. The last 2 of them are currently under investigation. PARPis are used in patients with recurrent ovarian cancer and BRCA mutation [58]. It seems that combined treatment with PARPis and immune checkpoint blockade might be beneficial for patients with BRCA mutation. A phase I study, in which olaparib and tremelimumab [cytotoxic T-cell antigen 4 (CTLA4) immune checkpoint antibody] were used, was conducted to assess this regimen's tolerability. No dose-limiting toxicities were identified [59]. Two more studies are currently exploring these treatment strategies. The first one is the combined therapy with niraparib and pembrolizumab [programmed death 1 (PD-1) checkpoint inhibitor] in patients with triple-negative breast cancer or recurrent ovarian cancer. The phase $1 / 2$ study results are promising, with an overall response rate (ORR) of $25 \%$ in platinum-resistant ovarian cancer and an ORR of $45 \%$ in patients with BRCA mutations. The most common reported side effects were anaemia and thrombocytopaenia [60]. Another study suggested a synergistic therapeutic effect of the combination of olaparib and durvalumab [anti-programmed cell death ligand-1 (PD-L1)] in patients with relapsed gastric cancer [61].

Several new cell cycle checkpoint inhibitors are currently under investigation. A recent study reported the efficacy of prexasertib - a second-generation checkpoint first and second kinase (CHK1/2) inhibitor for patients who are diagnosed with BRCA wild-type high grade serous ovarian cancer and who have undergone intensive treatment. Prexasertib seems to be a useful tool in treating patients with platinum-resistant or platinum-refractory disease [62]. The studies reported that the combination of PARPi with Ataxia Telangiectasia and Rad3-related (ATR) and its downstream Checkpoint Kinase 1 (CHK1) inhibitor is more effective in reducing tumour burden in BRCA mutation models, in comparison to monotherapy [63].

Folate receptor- $\alpha$ (FOLR-1), which is highly expressed in tumour tissue in HGSC patients and usually undetectable in normal tissue, is another potential therapeutic target [64]. FOLR-1 is a glycosylphosphatidylinositol-connected membrane glycoprotein, which is exposed to the extracellular molecules [65]. It is involved in DNA replication and damage repair in cells by mediating cellular responses to foliate, including proliferation, cell division, and tissue growth [66]. Some trials focused on monoclonal antibodies binding to the FOLR-1 - farletuzumab [67] and mirvetuximab soravtansine [68]. Moreover, the adoptive cell transfer (ACT) therapy, in which tumour-specific cytotoxic T-cells are expanded in vivo and then infused after lymphodepleting chemotherapy [69], might be used in EOC patients. Westergaard et al. expanded tumour-infiltrating lymphocytes (TILs) from 34 tumour specimens of ovarian cancer and showed the recognition of autologous tumour cells in $>50 \%$ of the patients. Moreover, antigen-specific TILs were isolated and further expanded in vivo. These findings supported the hypothesis that patients with OC could benefit from ACT [70] and were followed by the pilot study [71].

Cancer stem cells (CSCS) are a population of tumour cells with self-renewal abilities responsible for both tumour development and resistance to the applied treatment [72]. The drug resistance in CSCs leads to relapses during treatment [73]. Signalling pathways such as Wnt/ $\beta$-catenin and NOTCH are, among others, in charge of chemoresistance in CSCs [74, 75]. Therefore, inhibition of the Wnt pathway might become an efficient management in EOC treatment. Ipafricept (OMP-54F28), a firstin-class recombinant fusion protein with the extracellular part of human Frizzled-8 receptors fused to a human IgG1 Fc fragment that binds Wht ligands, is undergoing phase I study in pancreatic and ovarian cancers [76]. Additionally, some trials concentrate on Notch pathway silencing in EOC treatment. Delta-like ligand 4 (DII4), one of the Notch ligands, has been proven to be overexpressed in ovarian cancer. Dll4 is claimed, among others, to be responsible for tumour resistance to the anti-VEGF therapy [77]. The study by Huang et al. showed that combining DII4 inhibitors (murine REGN1035 and human REGN421) with anti-VEGF treatment (aflibercept) significantly reduces ovarian tumour growth. Dll4 blockade (REGN1035) combined with aflibercept has more significant therapeutic effects. This success may result from the increased apoptosis in tumour cells and increased transcription factor-GATA3 expression under hypoxia conditions [78].

\section{Conclusions}

Epithelial ovarian cancer is the most lethal gynaecological malignancy worldwide. It is usually diagnosed in the advanced stage and is associated with poor outcomes. Therefore, searching for and analysing the risk factors of these malignancies is extremely valuable. It can lead to the development of new prevention strategies and a decrease in cancer-related deaths. As the general principle says, prevention is better than cure. Recent years have brought reports about different genes and molecules, which may serve as prognostic factors for EOC patients. They can be used to stratify patients, decide about the usage of aggressive treatment strategies, or predict chemoresistance. Efforts are also being made to increase the clinical effectiveness of the treatment while minimizing toxicities for the patients. Several findings have identified potential new attractive therapeutic approaches, which are still under investigation. Recent studies suggest that EOC patients might benefit from immunotherapy.

The authors declare no conflict of interest.

\section{References}

1. Holschneider CH, Berek JS. Ovarian cancer: epidemiology, biology, and prognostic factors. Semin Surg Oncol 2000; 19: 3-10.

2. Oberaigner W, Minicozzi P, Bielska-Lasota M, et al. Survival for ovarian cancer in Europe: the across-country variation did not shrink in the past decade. Acta Oncol 2012; 51: 441-453. 
3. Eisenhauer EA. Real-world evidence in the treatment of ovarian cancer. Ann Oncol 2017; 28 (Suppl_8): 861-865.

4. Quaglia A, Tavilla A, Shack L, et al. The cancer survival gap between elderly and middle-aged patients in europe is widening. Eur J Cancer 2009; 45: 1006-1016.

5. Marth C, Hiebl S, Oberaigner W, Winter R, Leodolter S, Sevelda P. Influence of department volume on survival for ovarian cancer: results from a prospective quality assurance program of the Austrian association for gynecologic oncology. Int J Gynecol Cancer 2009; 19: 94-102.

6. Chan J, Urban R, Cheung M, et al. Ovarian cancer in younger vs. older women: a population-based analysis. Br J Cancer 2006; 95: 1314-1320.

7. Nagle C, Bain C, Green A, Webb P. The influence of reproductive and hormonal factors on ovarian cancer survival. Int J Gynecol Cancer 2008; 18: 407-413.

8. Stewart C, Ralyea C, Lockwood S. Ovarian cancer: an integrated review. Semin Oncol Nurs 2019; 35: 151-156.

9. Ovarian Cancer Statistics. 2008 [15.11.2020].

10. Jayson GC, Kohn EC, Kitchener HC, Ledermann JA. Ovarian cancer Lancet 2014; 384: 1376-1388.

11. Goff BA, Mandel LS, Melancon CH, Muntz HG. Frequency of symptoms of ovarian cancer in women presenting to primary care clinics. JAMA 2004; 291: 2705-2712.

12. Lheureux S, Gourley C, Vergote I, Oza AM. Epithelial ovarian cancer. Lancet 2019; 393: 1240-1253.

13. Fu Z, Moysich K, Ness RB, Modugno F. Gender of offspring and risk of ovarian cancer: the Hope study. Cancer Epidemiol 2020; 64: 1016-1046.

14. Gierach GL, Modugno F, Ness RB. Gender of offspring and maternal ovarian cancer risk. Gynecol Oncol 2006; 101: 476-480.

15. Baik I, Lambe M, Liu Q, et al. Gender of offspring and maternal risk of invasive epithelial ovarian cancer. Cancer Epidemiol Biomarkers Prev 2007; 16: 2314-23120.

16. Modugno F, Fu Z, Jordan SJ, et al. Offspring sex and risk of epithelial ovarian cancer: a multinational pooled analysis of 12 case-control studies. Eur J Epidemiol 2020; 35: 1025-1042.

17. Modugno F, Goughnour SL, Wallack D, et al. Breastfeeding factors and risk of epithelial ovarian cancer. Gynecol Oncol 2019; 153: 116 122

18. Kotsopoulos J, Gronwald J, McCuaig JM, et al. Breastfeeding and the risk of epithelial ovarian cancer among women with a BRCA 1 or BRCA 2 mutation. Gynecol Oncol 2020; 159: 820-826.

19. Guleria S, Jensen A, Toender A, Kjaer SK. Risk of epithelial ovarian cancer among women with benign ovarian tumors: a follow-up study. Cancer Causes Control 2020; 31: 25-31.

20. Huang T, Townsend MK, Wentzensen N, et al. Reproductive and hormonal factors and risk of ovarian cancer by tumor dominance: results from the ovarian cancer cohort consortium (OC3). Cancer Epidemiol Biomarkers Prev 2020; 29: 200-207.

21. Pathak S, Wilczyński JR, Paradowska E. Factors in oncogenesis: viral infections in ovarian cancer. Cancers (Basel) 2020; 12: 561.

22. Slomovitz B, de Haydu C, Taub M, Coleman RL, Monk BJ. Asbestos and ovarian cancer: examining the historical evidence. Int J Gynecol Cancer 2021; 31: 122-128.

23. Steffen JE, Tran T, Yimam M, et al. Serous ovarian cancer caused by exposure to asbestos and fibrous talc in cosmetic talc powders-a case Series. J Occup Environ Med 2020; 62: 65-77.

24. Javadi S, Ganeshan DM, Qayyum A, Iyer RB, Bhosale P. Ovarian cancer, the revised FIGO staging system, and the role of imaging. AJR Am J Roentgenol 2016; 206: 1351-1360.

25. Pavlik EJ, Smith C, Dennis TS, et al. Disease-specific survival of type I and type II epithelial ovarian cancers-stage challenges categorical assignments of indolence \& aggressiveness. Diagnostics (Basel, Switzerland) 2020; 10: 56.

26. Bookman MA, Brady MF, McGuire WP, et al. Evaluation of new platinum-based treatment regimens in advanced-stage ovarian cancer: a phase III trial of the gynecologic cancer intergroup. J Clin Oncol 2009; 27: 1419-1425.

27. Engelberth SA, Hempel N, Bergkvist M. Development of nanoscale approaches for ovarian cancer therapeutics and diagnostics. Crit Rev Oncog 2014; 19: 281-315.
28. Bélanger F, Fortier E, Dubé M, et al. Replication protein a availability during DNA replication stress is a major determinant of cisplatin resistance in ovarian cancer cells. Cancer Res 2018; 78: 5561-5573.

29. Xing L, Mi W, Zhang Y, et al. The identification of six risk genes for ovarian cancer Platinum response based on global network algorithm and verification analysis. J Cell Mol Med 2020; 24 9839-9852.

30. Zou R, Xu H, Li F, Wang S, Zhu L. Increased expression of UBE2T predicting poor survival of epithelial ovarian cancer: based on comprehensive analysis of UBE2s, DNA Cell Biol 2020; 40: 36-60.

31. Li J, Zhi X, Shen X, et al. Depletion of Ube2c reduces ovarian cancer malignancy and reverses Cisplatin resistance via downregulating Cdk1. Biochem Biophys Res Commun 2021; 523(2): 434-40.

32. Martínez-Canales S, López de Rodas M, Nuncia-Cantarero M, et al. Functional transcriptomic annotation and protein-protein interaction nalysis identify EZH2 and UBE2C as key upregulated proteins in ovarian cancer. Cancer Med 2018; 7: 1896-1907.

33. Dwivedi SKD, Shameer K, Dey A, et al. KRCC1: a potential therapeutic target in ovarian cancer. FASEB J 2020; 34: 2287-2300.

34. Gou R, Zhu L, Zheng M, et al. Annexin A8 can serve as potential rognostic biomarker and therapeutic target for ovarian cancer: based on the comprehensive analysis of Annexins. J Transl Med 2019; 17: 275.

35. Cao T, Pan W, Sun X, Shen H. Increased expression of TET3 predicts unfavorable prognosis in patients with ovarian cancer - a bioinformatics integrative analysis. J Ovarian Res 2019; 12: 101.

36. Wolf D, Fiegl H, Zeimet AG, et al. High Rig-I expression in ovarian cancer associates with an immune-escape signature and poor clinical outcome. Int J Cancer 2020; 146: 2007-2018.

37. Kim J, Beidler P, Wang H, et al. Desmoglein-2 as a prognostic and biomarker in ovarian cancer. Cancer Biol Ther 2020: 21: 1154-1162.

38. Ikeda Y, Sato S, Yabuno A, et al. High expression of maternal embryonic Leucine-Zipper Kinase (Melk) impacts clinical outcomes in patients with ovarian cancer and its inhibition suppresses ovarian cancer cells growth ex vivo. J Gynecol Oncol 2020; 31: 93

39. Czogalla B, Partenheimer A, Jeschke $U$, et al. $\beta$-arrestin 2 is a prog nostic factor for survival of ovarian cancer patients upregulating cell proliferation. Front Endocrinol (Lausanne) 2020; 11: 554-733.

40. Wang C, Wen A, Qiao J, Liu Y, Guo Y, Wang W. High expression of ring finger protein 126 predicts unfavorable prognosis of epithelial ovarian cancer. Med Sci Monit 2020; 26: 921370.

41. Orr B, Edwards RP. Diagnosis and treatment of ovarian cancer. He matol Oncol Clin North Am 2018; 32: 943-964.

42. Rossi L, Verrico M, Zaccarelli E, et al. Bevacizumab in ovarian cancer: a critical review of phase III studies. Oncotarget 2017; 8: 12389-12405.

43. Deptała A, Omyła-Staszewska J, Krajewska K. Leczenie ukierunkowane na cele molekularne u chorych na nowotwory narządu rodnego, w: onkologii i hematoonkologii. Via Medica, Gdańsk 2013, 359-379.

44. Bochum S, Berger S, Martens UM. Olaparib. Recent results. Cancer Res 2018; 211: 217-233.

45. Elattar A, Bryant A, Winter-Roach BA, Hatem M, Naik R. Optima primary surgical treatment for advanced epithelial ovarian cancer. Cochrane Database Syst Rev 2011; 2011: 65-75.

46. Ceppi L, Bardhan NM, Na Y, et al. Real-time single-walled carbon nanotube-based fluorescence imaging improves survival after debulking surgery in an ovarian cancer model. ACS Nano 2019; 13: 5356-5365.

47. Syed V. TGF- $\beta$ signaling in cancer. J Cell Biochem 2016; 117: 1279 1287.

48. Bai Y, Li LD, Li J, et al. A FXYD5/TGF- $\beta / S M A D$ positive feedback loop drives epithelial-to-mesenchymal transition and promotes tumor growth and metastasis in ovarian cancer. Int J Oncol 2020; 56: 301 314.

49. De Gramont A, Faivre S, Raymond E. Novel TGF- $\beta$ inhibitors ready for prime time in onco-immunology. Oncoimmunol 2017; 6: 1257453.

50.Zhang Q, Hou X, Evans BJ, VanBlaricom JL, Weroha SJ, Cliby WA LY2157299 Monohydrate, a TGF- $\beta$ R 1 inhibitor, suppresses tumor growth and ascites development in ovarian cancer. Cancers (Basel) 2018; 10: 260 
51. Liu YL, Zhou OC, lasonos A, et al. Pre-operative neoadjuvant chemotherapy cycles and survival in newly diagnosed ovarian cancer: what is the optimal number? A Memorial Sloan Kettering Cancer Center team ovary study. Int J Gynecol Cancer 2020; 30: 1915-1921.

52. Roelofsen T, Wefers C, Gorris MAJ, et al. Spontaneous regression of ovarian carcinoma after septic peritonitis; a unique case report. Front Oncol 2018; 8: 562.

53. Fujiwaki R, Sawada K. Spontaneous regression in recurrent epithelial ovarian cancer. Arch Gynecol Obstet 2007; 275: 389-391.

54. Oda K, Hamanishi J, Matsuo K, Hasegawa K. Genomics to immuno therapy of ovarian clear cell carcinoma: unique opportunities for management. Gynecol Oncol 2018; 151: 381-389.

55. Zhang L, Conejo-Garcia JR, Katsaros D, et al. Intratumoral T-cells, recurrence, and survival in epithelial ovarian. Cancer 2003; 348: 203-213.

56. Zamarin D, Burger RA, Sill MW, et al. Randomized phase II trial of Nivolumab versus Nivolumab and Ipilimumab for recurrent or persistent ovarian cancer: an oncology study. J Clin Oncol 2020; 38: 1814-1823.

57. Khan KA, Kerbel RS. Improving immunotherapy outcomes with anti-angiogenic treatments and vice versa. Nat Rev Clin Oncol 2018; 15: $310-324$

58. Taylor KN, Eskander RN. PARP inhibitors in epithelial ovarian cancer. Recent Pat Anticancer Drug Discov 2018; 13: 145-158.

59. Adams SF, Rixe O, McCance D, et al. Phase I study combining PARP-inhibition with immune checkpoint blockade in women with BRCA-deficient recurrent ovarian cancer. Gynecol Oncol 2017; 145: 99-100.

60. Konstantinopoulos PA, Waggoner SE, Vidal GA, et al. TOPACIO/ Keynote-162 (NCT02657889): a phase 1/2 study of Niraparib + Pembrolizumab in patients (pts) with advanced triple-negative breast cancer or recurrent ovarian cancer (Roc) - results from roc cohort. J Clin Oncol 2018; 36 (15 suppl): 106.

61. Drew Y, de Jonge M, Hong SH, et al. An open-label, phase II basket study of Olaparib and Durvalumab (Mediola): sesults in germline BRCA-mutated (G BRCA M) Platinum-sensitive relapsed (Psr) ovarian cancer (Oc). Gynecol Oncol 2018; 149: 246-247.

62. Lee JM, Nair J, Zimmer A, et al. Prexasertib, a cell cycle checkpoint kinase 1 and 2 inhibitor, in BRCA wild-type recurrent high-grade serous ovarian cancer: a first-in-class proof-of-concept phase 2 study. Lancet Oncol 2018; 19: 207-215.

63. Kim H, George E, Ragland R, et al. Targeting the ATR/CHK1 Axis with PARP inhibition results in tumor regression in BRCA-mutant ovarian cancer models. Clin Cancer Res 2017; 23: 3097-3108.

64. Köbel M, Madore J, Ramus SJ, et al. Evidence for a time-dependent association between Folr1 expression and survival from ovarian carcinoma: implications for clinical testing. An ovarian tumour tissue analysis consortium study. Br J Cancer 2014; 111: 2297-307.

65. Ragoussis J, Senger G, Trowsdale J, Campbell IG. Genomic organization of the human folate receptor genes on chromosome 11q13. Genomics 1992; 14: 423-430.

66. Sierra EE, Goldman ID. Recent advances in the understanding of the mechanism of membrane transport of folates and antifolates. Semin Oncol 1999; 26 (2 Suppl 6): 11-23.

67. Vergote I, Armstrong D, Scambia G, et al. A randomized, double-blind, placebo-controlled, phase III study to assess efficacy and safety of weekly Farletuzumab in combination with Carboplatin and Taxane in patients with ovarian cancer in first Platinum-sensitive relapse. J Clin Oncol 2016; 34: 2271-2278.

68. Moore KN, Vergote I, Oaknin A, et al. Forward I: a phase III study of Mirvetuximab Soravtansine versus chemotherapy in Platinum-re sistant ovarian cancer. Future Oncol 2018; 14: 1669-1678.

69. Houot R, Schultz LM, Marabelle A, Kohrt H. T-cell-based immunotherapy: adoptive cell transfer and checkpoint inhibition. Cancer Immunol Res 2015; 3: 1115-1122.

70. Westergaard MCW, Andersen R, Chong C, et al. Tumour-reactive T-cell subsets in the microenvironment of ovarian cancer. $\mathrm{Br} J \mathrm{Can}$ cer 2019; 120: 424-434.

71. Pedersen M, Westergaard MCW, Milne K, et al. Adoptive cell therapy with tumor-infiltrating lymphocytes in patients with metastatic ovarian cancer: a pilot study. Oncoimmunol 2018; 7: 1502905.

72. Jordan CT, Guzman ML, Noble M. Cancer stem cells. N Engl J Med 2006; 355: 1253-1261.
73. Valent $P$, Bonnet $D$, de Maria $R$, et al. Cancer stem cell definitions and terminology: the devil is in the details. Nat Rev Cancer 2012; 12: 767-775.

74. Reya T, Duncan AW, Ailles L, et al. A role for Wnt signalling in self-renewal of haematopoietic stem cells. Nature 2003; 423 : 409-414.

75. Abbaszadegan MR, Riahi A, Forghanifard MM, Moghbeli M. Wnt and Notch signaling pathways as activators for epidermal growth factor receptor in esophageal squamous cell carcinoma. Cell Mol Biol Lett 2018; 23: 42

76. Jimeno A, Gordon M, Chugh R, et al. A first-in-human phase I study of the anticancer stem cell agent Ipafricept (OMP-54F28), a decoy receptor for Wnt Ligands, in patients with advanced solid tumors. Clin Cancer Res 2017; 23: 7490-7497.

77. Hu W, Lu C, Dong HH, et al. Biological roles of the Delta family Notch ligand Dll4 in tumor and endothelial cells in ovarian cancer. Cancer Res 2011; 71: 6030-6039.

78. Huang J, Hu W, Hu L, et al. Dll4 inhibition plus Aflibercept markedly reduces ovarian tumor growth. Mol Cancer Ther 2016; 15: 1344-1352.

\section{Address for correspondence}

\section{Marta Magdalena Fudalej, MD}

Department of Cancer Prevention

Medical University of Warsaw

81 Żwirki i Wigury St.

01-091 Warsaw, Poland

e-mail:mmfudalej@gmail.com 